\title{
Morphological Integration of the Modern Human Mandible during Ontogeny
}

\author{
Joshua M. Polanski \\ Department of Math \& Science, Kirkwood Community College, Iowa City Campus, Iowa City, IA 52240, USA \\ Correspondence should be addressed to Joshua M. Polanski, joshua.polanski@kirkwood.edu
}

Received 16 September 2010; Revised 3 January 2011; Accepted 24 February 2011

Academic Editor: Darren Curnoe

Copyright ( 2011 Joshua M. Polanski. This is an open access article distributed under the Creative Commons Attribution License, which permits unrestricted use, distribution, and reproduction in any medium, provided the original work is properly cited.

Craniofacial integration is prevalent in anatomical modernity research. Little investigation has been done on mandibular integration. Integration patterns were quantified in a longitudinal modern human sample of mandibles. This integration pattern is one of modularization between the alveolar and muscle attachment regions, but with age-specific differences. The ascending ramus and nonalveolar portions of the corpus remain integrated throughout ontogeny. The alveolar region is dynamic, becoming modularized according to the needs of the mandible at a particular developmental stage. Early in ontogeny, this modularity reflects the need for space for the developing dentition; later, modularity is more reflective of mastication. The overall pattern of modern human mandibular integration follows the integration pattern seen in other mammals, including chimpanzees. Given the differences in craniofacial integration patterns between humans and chimpanzees, but the similarities in mandibular integration, it is likely that the mandible has played the more passive role in hominin skull evolution.

\section{Introduction}

Morphological integration of the cranium has become a dominant research paradigm in biological anthropology. While it began with the early work of Weidenreich $[1,2]$ and the functional matrix model of Moss [3, 4], morphological integration became a driving force in anthropological research through work on macaques and New World monkeys by Cheverud and colleagues [5-11]. Building upon this earlier work, recent years have seen new techniques defined to quantify and assessing morphological integration being used to address the question of hominin evolution, with a particular emphasis on the evolution of modern humans [12-19].

In virtually all hominin studies involving morphological integration, the cranium serves as the major anatomical unit under investigation. The reasons for this are obvious. Craniodental remains are the most predominant morphology in the hominin and primate fossil records; also, taxonomic and evolutionary relationships tend to be defined primarily by cranial morphology. Because of this, a well-defined understanding of the relationships between cranial elements in both living and fossil forms is of extraordinary use. However, mandibular remains are also prevalent within the fossil record. The question then must be asked: why has the mandible been left behind in the field of hominin morphological integration? The mandible serves as an integral component of the skull and has been used extensively in numerous anthropological research questions. Mandibles have served, at one time or another, as type specimens for various hominin species: Australopithecus anamensis [20]; A. afarensis [21]; A. bahrelghazali [22]; Paranthropus crassidens [23]; Homo ergaster [24]; H. heidelbergensis [25].

Although a complete survey of the entire biomechanical anthropological literature is beyond the scope of this paper, the mandible has served as the backbone for numerous studies on biomechanics and stress-loading studies in both hominins and the rest of the primate order, particularly in, but not limited to, the work of Hylander and colleagues [2631]. The mandible has also been analyzed in relation to the evolutionary history of the chin and questions concerning its utility as a defining characteristic of human anatomical modernity [32-38]. The mandible and its associated dentition have also factored into investigations into the dietary 
adaptations of primates from the great apes [39-42] to the creation of the "gracile" and "robust" clades of the australopiths [43-49].

Outside of hominin paleontology, morphological integration of the mandible has been studied in mice [5052], baboons [50] and chimpanzees and gorillas [53]. Given the emphasis on cranial form in the evolution of modern humans, and the extensive literature concerning nonhuman mandibular integration, the decreased focus of morphological integration research in hominins involving the mandible is surprising. One of the defining features of modern human evolution has been the marked decrease in facial prognathism [17, 54-57], a trend which is mirrored in the evolution of the mandible. Despite changes in the hominin face and the associated alterations of the mandibular form, little to no research into the patterns of integration in the mandible has been undertaken. Also, evolutionary biology has begun to understand the need to investigate evolution from a developmental perspective (evo-devo). This trend is reflected in biological anthropology with the inclusion of ontogeny in various studies, including those involving morphological integration $[12,17,19]$. This paper sets out to rectify this situation by investigating morphological integration of the mandible during ontogeny. More specifically, it will test the null hypothesis of no change in the pattern of integration in the mandible from early childhood to adulthood.

\section{Materials and Methods}

The modern human sample used in this analysis was derived from the Iowa Facial Growth study, created by and housed at the University of Iowa Dental School. The Iowa Facial Growth Study ran from 1946 to 1960 and was a true longitudinal study; after the original volunteer group reached age five no new participants were allowed into the study. Several years after the end of the Iowa Facial Growth study, participants were asked to be radiographed once more to study the facial skeleton after growth had ceased [58]. True longitudinal studies have an advantage over cross-sectional studies in that the data gathered from longitudinal studies allows for the study of actual growth; cross-sectional studies must use approximations of growth and can risk missing or excluding certain trends. However, longitudinal studies often suffer from a drain of available participants throughout the length of the study.

The subjects were ninety-seven percent Caucasian, all United States-born. Over ninety percent had at least threequarters of their grandparents descended from northern European immigrants (British Isles, Germany, France, Scandinavia, or The Netherlands), while the remainder possessed at least two grandparents from either central or southeast Europe. Subjects' families were of above average socioeconomic status; over fifty percent had fathers who worked in managerial/professional vocations, forty percent had fathers who owned small businesses or were skilled traders, and the remaining ten percent came from families where the fathers engaged in semiskilled trade [58].

The major criterion for inclusion in the Iowa Facial Growth Study was neither ethnic nor economic; the researchers were interested in subjects who lived in and around Iowa City at the beginning of the study and whose families were likely to remain in the same area for the duration of the subjects' development, as well as their willingness (or their parents' willingness) to participate in a long-term study. All subjects were voluntary ones, no subjects were compensated for their participation [58]. While the primary criterion was not dental occlusion, subjects with nonnormal occlusions were not included in the study. The nearly unanimous Caucasian nature of the participants, as well as the above average socioeconomic status, is likely the result of the influence that the University of Iowa (then the State University of Iowa) exerted on the demographics of Iowa City in the mid-1940s.

The Iowa Facial Growth Study consists of radiographs taken in both norma lateralis and norma frontalis; because lateral radiographs are more commonly used in anthropological and developmental studies, only the lateral radiographs were utilized in this particular analysis, allowing for comparison of these results to those in other studies. These lateral and posteroanterior radiographs were originally taken at three-month intervals until the subjects reached age five, after which they were taken semiannually until age twelve, when the radiographs were taken annually. Subjects were examined, once the annual examinations began, within five days of their birthdays.

The sample used in this study is a mixed-sex sample. The Iowa Facial Growth Study was large at its inception, but shrunk precipitously over its run; not enough individuals are in the study at the end to allow for separation of males and females. Rather than focus on determining the pattern of integration at every stage of growth, this study instead decided to focus on the pattern of integration at the beginning of mandibular growth (or at the very least the youngest age available in the Iowa Facial Growth study), the pattern of integration at the onset of puberty, and the pattern of mandibular integration after the cessation of mandibular growth.

The sample in this study consisted of 70 individuals (36 males, 34 females) drawn from the Iowa Facial Growth Study. Criteria were only that the individuals had completed the entire study and had returned to take place in the latter follow-up study. Three developmental stages were created. Group no. 1 consisted of radiographs of the 70 individuals from the time period when the subjects were between the ages of 4 and 5 and one-half years old. Group no. 2 consisted of radiographs of those same 70 individuals from the time period when they were between 10 and 12 years old. Group no. 3 consisted of radiographs of those same individuals taken from the time period when they were between 20 and 29 years of age. Only one lateral radiograph from any one subject was used in each grouping, that is, in group no. 1, if one individual had radiographs at four years, four and one half years, five years, and so forth, the best quality radiograph was chosen.

The developmental groups should encompass most of the postbirth mandibular growth, while not overlapping enough that certain groups would be more similar in their integration patterns because they have near-overlapping 
TABLE 1: Landmarks and derived measurements.

\begin{tabular}{lcc}
\hline Variable & Measurement & Abbr. \\
\hline Mandibular length & Condylion-gnathion & Cd-Gn \\
Ascending ramus height & Condylion-gonion & Cd-Go \\
Corpus length & Gonion-pogonion & Go-Pg \\
Upper mandibular length & Condylion-infradentale & Cd-I \\
Anterior corpus height & Menton-infradentale & Me-I \\
\hline
\end{tabular}

ages. While the developmental groups do have different numbers of years encompassed within them, this should not adversely affect the results. The groups are distant enough in time to encapsulate different periods of growth (i.e., 1st molar, 2nd molar, etc.). Also, because this is a true longitudinal study, any differences between these groups are likely to be reflective of real growth, rather than an a combination of different age ranges involving samples from different populations.

Six osteometric landmarks were located on the lateral radiographs for all individuals in all three developmental groups, and from these landmarks five standard mandibular linear measurements were taken (Table 1; Figures 1(a), 1(b), and $1(\mathrm{c})$ ). Because the proposed research deals with modern humans at different ontogenetic stages, the issue of landmark and linear measurement repeatability must be addressed. The most important criterion for choosing measurements in an ontogenetic study is repeatability between age or developmental groups [59]. To avoid potential difficulties, only skeletal landmarks and derived linear measurements that could be found at all stages throughout ontogeny were selected; these measurements were taken from human mandibular developmental literature. The measurements were chosen to encompass the entire mandible without being overly redundant in any one dimension (height or length), as this could skew the results, making certain measurements from the same dimension appear more integrated than they are in reality $[18,19]$.

It should be noted that incision (I) is generally taken at the tip of the medial incisor. However, as the incisors at the beginning of this study are deciduous, and those at the end are adult, this point would not be truly homologous. Instead, incision is taken at the upper margin of the alveolar bone directly in front of the incisor (sort of a mandibular prosthion); this renders this landmark homologous.

These criteria do limit the number of available measurements. Standard metrics of the mandible can include condylion-gnathion, condylion-pogonion, condylion-point $\mathrm{B}$, all of which are considered mandibular length and all of which are redundant to one another to varying degrees. The number of measurements in this study is small; however, the measurement set encompasses mandibular variation and over-redundancy was avoided.

This paper also uses upper mandibular length as a proxy for true alveolar length. Determining the posterior end of the dental arcade so that it may include all the developing and erupting dentition, but none of the ramus, was not something that could be done and replicated on all individuals at all developmental stages. As such, true alveolar length would not be homologous across all individuals, and upper mandibular length was substituted as a reasonable, if imperfect, proxy.

As this study involves deriving linear measurements from radiographs, issues of parallax and magnification must be addressed. Parallax was minimized in the Iowa Facial Growth Study through the combination of a long distance between the skull of the subject and the anode (X-ray source) and a short distance between the subject's skull and the X-ray film; additionally, this same combination is what minimizes enlargement in these radiographs $[60,61]$. In the few instances where significant parallax existed and became an issue, midpoint averaging techniques were employed to create an average of the right and left sides of the mandible as they would have appeared in the midsagittal plane $[60,61]$.

Normally when dealing with linear measurements taken on radiographs magnification must be taken into account; the most common solution is to determine the percent of magnification which exists in the radiograph collection and scale the linear measurements accordingly. Because morphological integration in this study is quantified using conditional independence modeling (via correlation factors), this step is unnecessary. All radiographs in age group no. 1 have the same percent magnification, as do all the radiographs in age group no. 2 and age group no. 3, respectively. Because of this, all the measurements in each age group are scaled by the same factor (percent magnification) and therefore the correlations are the same whether the magnification is corrected for or not. As such, it is unnecessary to correct for magnification as magnification does not play a role in influencing the results of the analysis.

The sample utilized in this study is a mixed-sex sample; mixed-sex samples are nothing new in anthropology, and make up quite a number of the morphological integration literature $[6,10,12,13,18,19]$. While various methodologies exist to deal with sexual dimorphism in linear measurements for primates, the most commonly applied methodology when studying morphological integration is the method of "scaling" one sex so that its measurements more closely resemble those of the other sex. Using this method, an average value for each measurement for both sexes was calculated, and the difference between the male and female averages for each measurement was derived. This difference was then subtracted from of the male raw values for each measurement for every individual male; in a sense, the males have been scaled to be females $[6,10,18,19]$. Modern humans possess a low degree of sexual dimorphism, however, and the analysis was run on both raw (unscaled) and scaled (to resemble females) data; differences in the pattern of integration were negligible, but the results of the scaled data are presented here.

This scaling technique should also alleviate any potential puberty issues in developmental group no. 2 of the sample. While females enter puberty, on average, at younger ages than males, the male sample has been scaled up to be females in puberty. The females sample are all of the same general background and scioeconomic status [58], and as such are likely entering puberty at roughly the same time; slight differences in the timing between individual 


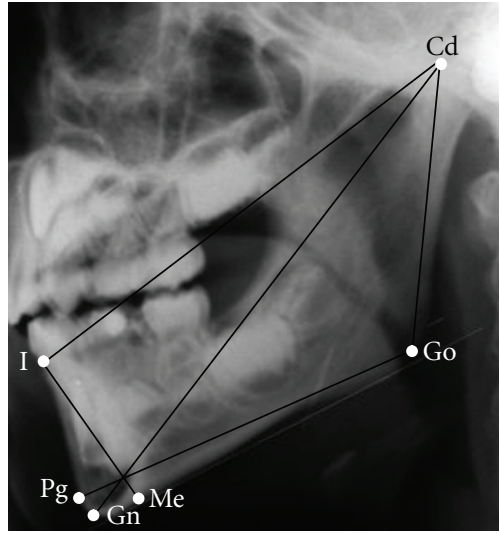

(a)

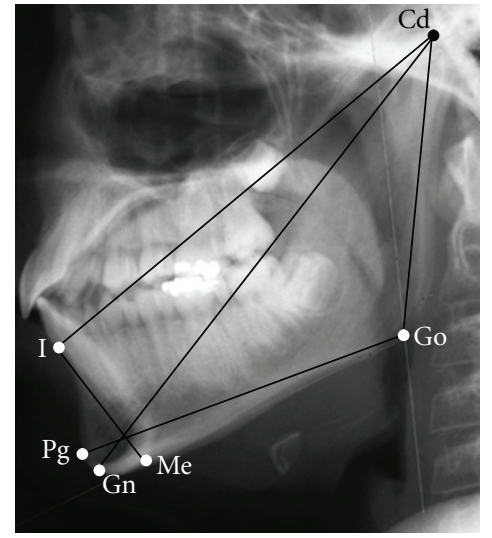

(b)

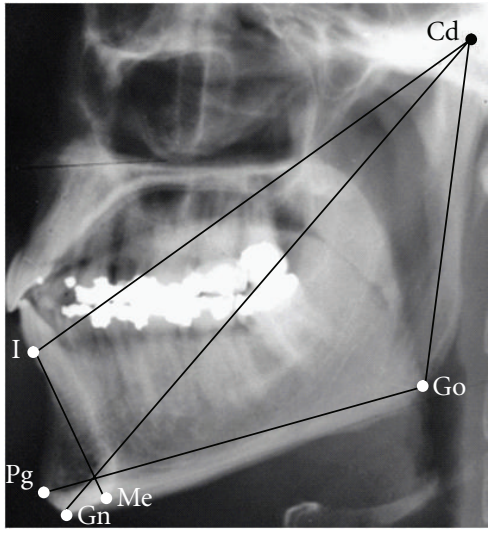

(c)

Figure 1: Landmarks and linear measurements. (a) Group no. 1. (b) Group no. 2. (c) Group no. 3.

females in the commencement of puberty is unlikely to have a deleterious effect on the analyses of group no. 2 . Numerous methodologies exist which have been used to investigate morphological integration $[6,8,15,51,59,62-$ 64]. Conditional independence modeling was chosen for this study because it has the ability to quantify both the pattern and relative degree of morphological integration in systems. Conditional independence modeling operates on the assumption of relative independence between systems; that is, nonintegrated (or modularized) systems are defined as possessing no statistically significant interaction between component variables, in this case linear measurements. The interaction between any two measurements in this study is referred to as an "edge," that is, the edge between mandibular length and corpus length or the edge between anterior corpus height and upper mandibular length.

In order to calculate edge-exclusion values, first a standard raw correlation matrix $(S)$ is converted into a "concentration" matrix $(\Omega)$ via matrix inversion $\left(\Omega=S^{-1}\right)$. Following this initial step, the concentration matrix is rescaled, such that the diagonal elements equal 1.0, as in a normal correlation matrix, while the off-diagonal elements are equal to the negatives of the partial correlation coefficients for all the pairwise variables. The following formula can be used to calculate the partial correlation coefficients:

$$
P_{i j \cdot[K]}=-\frac{\Omega_{i j}}{\left(\Omega_{i i} \Omega_{j j}\right)^{0.5}} .
$$

An edge is tested for statistical significance via the calculation of an "edge-exclusion value," which ranges from zero (complete modularity) to infinity (total integration). While edge-exclusion values can theoretically range to infinity, a brief survey of the literature demonstrates that most values do not exceed $180[18,19,65]$. Edge-exclusion values are calculated from these scaled partial correlation values, using the formula

$$
-N \cdot \ln \left(1-P_{i j \cdot[K]}^{2}\right)
$$

in which $N$ is the sample size for each group being tested, in this case the number of individuals in each of the three groups. Each edge-exclusion value is tested for statistical significance against a fully integrated asymptotic $\chi^{2}$ distribution, with one degree of freedom. This yields a methodologically determined edge-exclusion cutoff value of $3.84[18,19,65]$.

Edge-exclusion values below 3.84 are not statistically significantly integrated and therefore are modularized, while edge-exclusion values above 3.84 are statistically significantly integrated. If an edge is integrated, then the relationship between the two measurements that comprise it is integrated; if the edge is modularized, the relationship between the measurements is modularized. Additionally, edge-exclusion values determine the relative strength of interaction (integration) between any two measurements; values closer to zero are more modularized, whereas an edge-exclusion value of 100 indicates stronger morphological integration than a value of 4.3 , though both are statistically integrated $[18,19,65]$.

Besides possessing a methodologically determined statistically significant cutoff value for determining integration, conditional independence modeling also differs from other more common integration methodologies in that it requires no a priori need to assign measurements into functional units (i.e., the corpus and the ascending ramus of the mandible). And while conditional independence modeling is designed to quantify only the degree of interaction between any two variables, by studying the overall pattern of edge-exclusion values, it is possible to determine if different functional units (F-sets) are integrated; strong interunit integration would be indicated by integration of the edge-exclusion values for measurements from the different F-sets [18].

\section{Results}

Before detailing differences and similarities in the patterns of integration between the three developmental groups, it is advantageous to compare the overall level of integration between juvenile, adolescent, and adult modern human 
TABLE 2: Edge-exclusion values group $1^{1}$.

\begin{tabular}{lcccc}
\hline & Cd-Gn & Cd-Go & Go-Pg & Cd-I \\
\hline Cd-Go & $\mathbf{4 2 . 0 7}$ & - & & \\
Go-Pg & 37.5 & 44.1 & - & \\
Cd-I & $\mathbf{2 5 . 7}$ & 0.15 & 0.12 & - \\
Me-I & 0.007 & $\mathbf{9 . 6 0}$ & $\mathbf{6 . 6 4}$ & 1.04 \\
\hline Bold values are statistically significantly integrated. See text for explanation \\
of cutoff value.
\end{tabular}

TABLE 3: Edge-exclusion values group $2^{1}$.

\begin{tabular}{lcccc}
\hline & Cd-Gn & Cd-Go & Go-Pg & Cd-I \\
\hline Cd-Go & $\mathbf{3 7 . 2 5}$ & - & & \\
Go-Pg & $\mathbf{2 1 . 2 9}$ & $\mathbf{1 9 . 0 6}$ & - & \\
Cd-I & $\mathbf{4 0 . 0 3}$ & 0.44 & 0.43 & - \\
Me-I & 0.66 & 1.89 & 0.22 & 0.043 \\
\hline
\end{tabular}

${ }^{1}$ Bold values are statistically significantly integrated. See text for explanation of cutoff value.

TABLE 4: Edge-exclusion values group $3^{1}$.

\begin{tabular}{lcccc}
\hline & Cd-Gn & Cd-Go & Go-Pg & Cd-I \\
\hline Cd-Go & $\mathbf{2 9 . 5 1}$ & - & & \\
Go-Pg & $\mathbf{2 4 . 1 1}$ & $\mathbf{2 6 . 2 5}$ & - & \\
Cd-I & $\mathbf{1 1 . 4 9}$ & 3.02 & $\mathbf{7 . 1 0}$ & - \\
Me-I & $\mathbf{7 . 7 5}$ & 0.05 & 0.005 & 0.18 \\
\hline
\end{tabular}

${ }^{1}$ Bold values are statistically significantly integrated. See text for explanation of cutoff value.

mandibles. When edges are modularized in the developmental groups, the edge-exclusion values tend to be near zero, indicating strong separation of the regions (Tables 24). However, when edges are integrated, there is disparity between the two groups. Juvenile and adolescent modern humans have larger edge-exclusion values for their integrated edges than do the adults, meaning that when integration occurs, it is stronger in the earlier developmental stages than in adults (Tables 2-4). However, edge-exclusion values are designed for testing whether specific interactions between variables are statistically significant, and less for determining a general overview of integration. It has been demonstrated elsewhere $[12,13,18]$ that the patterning of integration versus modularity is more informative than the overall level; as such, the pattern of integration shall de detailed and discussed in-depth.

3.1. Group No. 1 (4-5 Years). The overall pattern of integration in this age group (Table 2) is one of the strongly integrated edges in certain regions and strongly modularized edges in others. Mandibular length is characterized mainly by integration in its edge-exclusion values. Strong integration can be seen in the edges between mandibular length and ascending ramus height and corpus length; the former edge is characterized by a slightly more robust degree of integration. Moderate morphological integration is seen in the edgeexclusion value for the mandibular length edge involving upper mandibular length. Modularity is seen only in the edge-exclusion value for the edge between mandibular length and anterior corpus height; this edge displays very strong modularity in its edge-exclusion value.

Integration characterizes the majority of the edges involving ascending ramus height. In particular, strong integration exists between this measurement and both mandibular length and corpus length, as can be seen in the respective edge-exclusion values. The edge-exclusion value for the edge between ascending ramus height and anterior corpus height displays fairly weak integration. Modularity in the edges involving ascending ramus height is found only in the edge with upper mandibular length, with an edge-exclusion value indicating that the modularity found here is quite robust.

Corpus length is characterized by integration in most of its edges. Strong integration shows up in the edge-exclusion values for the corpus length edges involving mandibular length and ascending ramus height; the edge-exclusion value for the corpus length to ascending ramus height edge is the more integrated of the two. A weakly integrated edge-exclusion value characterizes the edge between corpus length and anterior corpus height. Finally, the edge between corpus length and upper mandibular length shows strong modularity in its edge-exclusion value, the only modularized edge involving corpus length.

Modularity makes up the majority of the edges involving upper mandibular length. Integration can only be seen in the edge-exclusion value for the edge involving mandibular length; the edge displays moderate integration in its edgeexclusion value. The edge-exclusion values for the edges between upper mandibular length and ascending ramus height and corpus length both are characterized by strong modularity, with near identical edge-exclusion values. More moderate modularity is seen in the edge-exclusion value between upper mandibular length and anterior corpus height.

Anterior corpus height demonstrates an even split between modularity and integration. Integration can be seen in the edges involving ascending ramus height and corpus length; the edge-exclusion values for both edges indicate weak integration, with the former edge being slightly more integrated than the latter. The edge between anterior corpus height and mandibular length has a strongly modularized edge-exclusion value. Moderate modularity exists in the edge between anterior corpus height and upper mandibular length.

3.2. Group No. 2 (10-12 Years). As with group no. 1, group no. 2 is characterized by strong integration in certain regions and strong modularity in others (Table 3). Mandibular length has strongly integrated edges with ascending ramus height, corpus length, and upper mandibular length. It is strongly modularized in its relationship with anterior corpus height. Ascending ramus height is integrated strongly in its edges with mandibular length and corpus length, but shows modularity in the edges involving upper mandibular length and anterior corpus height, with the former the more modularized of the two edge-exclusion values. Corpus length is integrated relatively strongly in the edges involving mandibular length and ascending ramus height, but is 
modularized strongly in its relationship to upper mandibular length and anterior corpus height. Upper mandibular length is integrated only with mandibular length, and this relationship between these two measurements is characterized by strong integration; its edge-exclusion values with the remaining measurements are all strongly modularized. Finally, anterior corpus height shows complete modularity with the other measurements, with none of its edge exclusion values being integrated.

3.3. Age Group No. 3 (20-29 Years). The adult mandible has strongly modularized edges in certain regions, while its integrated regions do not show the strength of association as was seen in the earlier groups (Table 4). Mandibular length displays integration in all of its associated edges; however, the degree of integration for these edges does differ. The edge-exclusion values for the mandibular length to ascending ramus height edge and the mandibular length-corpus length edge both display stronger integration than is seen in the edges with upper mandibular length and anterior corpus height.

Ascending ramus height possesses an equal number of both integrated and modularized edges. Moderate integration characterizes the edge-exclusion values for edges involving both mandibular length and corpus length. Modularity characterizes the remaining edges, although differences exist in the strength of this modularity. The mandibular length to upper mandibular length edge has very weak modularity in its edge-exclusion value; this edge-exclusion value is quite near the methodologically determined cutoff value (3.84) for being statistically significantly integrated. This likely is indicative of a biologically important relationship, even though it does not achieve true statistical significance. The remaining edge, mandibular length to upper mandibular length, has a strongly modularized edge-exclusion value.

Corpus length is characterized by mainly integrated edges, which display varying degrees of strength. Moderate integration can be found in the edge-exclusion values for both the corpus length to mandibular length and the corpus length to ascending ramus height edges. Weaker integration exists in the corpus length to upper mandibular length edge, as evidenced by its edge-exclusion value. Modularity only exists in the relationship between corpus length and anterior corpus height; the edge-exclusion value is indicative of strong modularity.

Upper mandibular length shows a mixture of integrated and modularized edges. Integration is seen in the edges involving mandibular length and corpus length. The edges involving mandibular length and corpus length are both integrated, although the former has a slightly stronger relationship. The edge between upper mandibular length and ascending ramus height, as mentioned above, is weakly modularized. Finally, the edge involving anterior corpus height shows strong modularity in its edge-exclusion value.

Anterior corpus height is characterized by modularity. Only the edge between anterior corpus height and mandibular length is integrated; its edge-exclusion value indicates weak integration. Modularity defines the remaining edges.
All (ascending ramus height, corpus length, upper mandibular length) are characterized by strong modularity; the least modularized edge involves upper mandibular length, while the most modularized edge is with corpus length.

\section{Discussion}

The overall degree of integration seen in the modern human mandible during ontogeny decreases, with integrated edges possessing lower edge-exclusion values, indicating weaker integration, in the adult group than in either the juvenile or adolescent groups.

More important than the overall level of integration is the change in the pattern of integration seen across ontogeny. Juvenile modern humans (Figure 2(a)) are characterized by strong integration between the posterior and inferior regions, exhibited by the strongly integrated edge exclusion value between the ascending ramus height and corpus length. Further, there is a weaker but meaningful amount of integration between these two regions and the anterior portion of the mandible, seen in the edge-exclusion values for the edge involving anterior corpus height and ascending ramus height and corpus length. Finally, the juvenile mandible shows separation (or modularity) between the superior aspect and the anterior, posterior and inferior regions of the mandible; this separation is demonstrated by the upper mandibular length's modularity in its edges with all these measurements.

While the adolescent group (Figure 2(b)) is characterized by an overall increase in the number of modularized edges, it shares the pattern seen in the juvenile group of strong integration between the posterior and inferior margins of the mandible and separation of upper mandibular length from the other aspects of the mandible. The major differences between these two stages of ontogeny can be seen in the lack of integration between anterior corpus height and ascending ramus height and corpus length in the adolescent stage of growth.

Like the patterns seen in the preceding ontogenetic stages, the adult modern human mandible (Figure 2(c)) shows integration in the relationships between the posterior (ascending ramus height) and inferior (corpus length) regions. Despite these similarities, the adult modern human mandible differs from both the juvenile and adolescent mandibular patterns of integration in several ways. Unlike the juvenile and adolescent stages, the adult stage is characterized increased integration of upper mandibular length in its edges with other portions of the mandible. This can be seen in the relatively weakly integrated edge-exclusion value between upper mandibular length and corpus length.

Additionally, the upper mandibular length is extremely weakly modularized in its relationship with ascending ramus height; this edge-exclusion value near the methodologically determined cutoff value for statistical significance indicates that while this relationship is not statistically significant, it is likely biologically meaningful and can therefore be viewed as integrated. The adult stage of growth for modern humans differs from the juvenile stage in also being modularized in the edges involving anterior corpus height, specifically its edges with ascending ramus height and corpus length. 


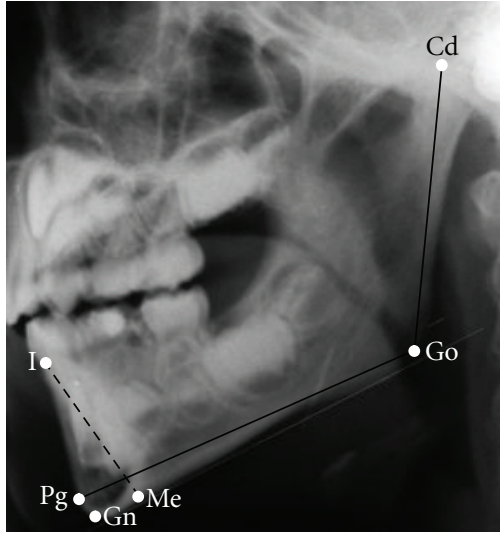

(a)

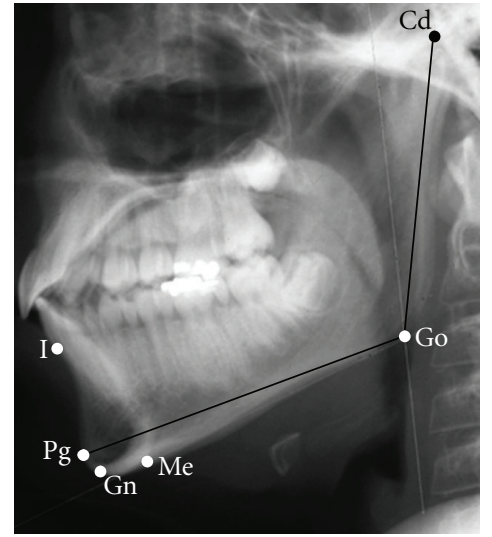

(b)

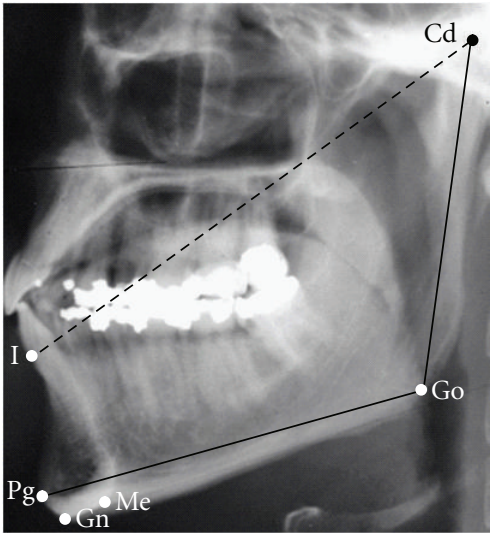

(c)

FIGURE 2: Integrated measurements. Lines indicate measurements whose edges are integrated with one another. A dashed line indicates weaker integration of that measurement with the others shown. See Figure 1 for explanation of (a), (b), and (c) groupings.

Overall, human mandibular ontogenetic integration can be characterized as maintained integration between the posterior and inferior portions of the mandible, paired with an increasing amount of modularity of the anterior portion of the corpus and an increasing amount of integration of the upper mandibular length with the remainder of the mandible.

What is the likely cause of this change in integration pattern throughout ontogeny? It is important to remember that while the mandible is often regarded as one monolithic structure, it does in fact serve two purposes, albeit both related to the same overall function: mastication. First, the mandible serves as the structure for the attachment of the chewing musculature: the temporalis (ascending ramus) and the portions of the medial pterygoid and masseter muscles (corpus length). Secondly, the mandible houses both the developing and erupted dentition [50, 51, 66, 67]. It is likely that the interaction of these two primary purposes shapes the patterns of mandibular integration seen in both throughout ontogeny.

The modern human mandible displays integration between the ascending ramus height and the corpus length in all of the quantified ontogenetic stages. Both these mandibular structures serve as attachment sites for various chewing musculatures, musculatures that must function together in order for the mandible to work properly. Despite overall changes in the size of the mandible throughout ontogeny, the primary function of the chewing muscles is not altered; they raise and lower the mandible and must do so in concert with one another. In addition to their shared function, these two regions also arise from the same embryonic stem cells [68]. As such, an establishment of a well-integrated relationship between these two regions early on in ontogeny makes sense, as does the retention of this integrated relationship throughout childhood and into adulthood. Predictably, this is what is seen in the patterns of integration in the results of this study.

Changes in the pattern of integration between juvenile and adolescent and adult modern human stages are primarily found in the relationships of upper mandibular length and anterior upper mandibular length shows strong modularization from the remaining mandibular measurements, implying that this area exists as a separate morphological module at these stages of development. Given what this measurement encompasses, the length of the alveolar portion of the corpus, it is likely that this separation is related to aspects of dentition.

At the juvenile and adolescent stages of development the human mandible is undergoing numerous changes related to the dentition. The mandible requires enough length to house all the dentition, both developing and erupted, during this period. Specifically, it needs to be large enough to house all the human deciduous dentition, as well as the developing forms of that same dentition and the buds of two new molars and eventually a third [69] in various stages of development or eruption. All of this must happen while allowing the chewing muscles to continue to function properly. As such, the separation of the alveolar portion of the mandible from those structures which serve as attachment sites for muscles makes sense; it would allow for the continued proper muscle function while at the same time ensuring there is enough flexibility for the developing dentition. The alveolus contains odontogenic cells not seen in the corpus [66], an embryological division between these two areas that is likely reflected in the increased modularity between them throughout ontogeny.

Anterior corpus height involves aspects of the alveolar region, as does upper mandibular length. The relationship between anterior corpus height and ascending ramus height and corpus length is integrated in the juvenile ontogenetic stage. In both the adolescent and adult stages, anterior corpus height is modularized within its edges with those two measurements. Anterior corpus height encompasses the alveolar bed, where dentition develops prior to eruption. In Group no. 1 (4-5 years), the tooth buds for most of the adult teeth are still forming in the alveolar bone, while in Group no. 2 (10-12 years) only the third molars are still forming and most of the adult dentition has erupted, and in Group no. 3 (20-29 years) all dentition has erupted [69]. If tooth buds sense a lack of room, they either fail to develop or become 
impacted [70-73]. It may be that the integration of anterior corpus height in its edges in the juvenile stage is indicative of a need of cooperation within the mandible to ensure proper dental development. Conversely, once all adult teeth have developed, as in the latter stages, this integrated relationship is no longer necessary, and so anterior corpus height becomes modularized in its edges with the other measurements, and the alveolar bed separates from the rest of the mandible.

If the integration of the edges involving anterior corpus height with the measurements representing other portions of the mandible in juveniles is related to dental development, the modularization of this area in adolescents and adults may be related to occlusion. The mandible needs to be high enough so that the dentition can occlude with its corresponding maxillary dentition correctly. If this is the case, then it would be expected that anterior corpus height would integrate with portions of the maxilla in modern humans. It is worth noting that the aspects of the lower maxilla in modern humans from this same Iowa Facial Growth Study are more modularized in adults than in juveniles [19]. This result could support this notion of increased modularity due to the need for occlusion, and is an avenue of research worth exploring.

Upper mandibular length in adults has become more integrated in its edges with most of the other measurements. The edge with corpus length has become statistically significantly integrated. The relationship between upper mandibular length and ascending ramus height also has changed. In juveniles and adolescents, these two measurements are strongly modularized in their edge; in adults, this relationship is still modularized, but very weakly. As such, it is likely that the upper mandibular length-ascending ramus height edge is biologically meaningful, although not statistically significant. It is possible, however, that this increase in integration between upper mandibular length and corpus length and ascending ramus height is influenced by upper mandibular length incorporating aspects of the ascending ramus. Given that upper mandibular length is modularized in its edges from corpus length and ascending ramus height in earlier ontogenetic stages (but still incorporates those same areas of the ramus) this is unlikely, but it cannot be completely discounted either.

Additionally, anterior corpus height is weakly integrated in its edge with overall mandibular length, a relationship not seen in the earlier ontogenetic stages. These relationships together in the adult stage indicate that, once dental development and eruption are complete [69] and mastication becomes the primary function of the mandible, the mandible becomes a weakly integrated superstructure, with strong aspects of modularity contained within.

While the juvenile stage in human mandibular ontogeny is characterized by a pattern of integration favoring dental development and the adult stage by a pattern characterized by chewing, the adolescent stage is characterized by an increase in overall modularity and the maintenance of several edges that are integrated throughout ontogeny. The adolescent stage marks the beginning of puberty in humans, a time of marked skeletal growth. It may be that this increase in modularity is necessary to allow the mandible to be flexible enough to function during a time of rapid skull growth. This same phenomenon is seen in the human cranium during ontogeny. Starting at the developmental stage that encapsulates puberty there is an increase in the amount of modularity seen in the cranium, with the retention of a few key relationships that are always integrated. This increased modularity in the cranium ends at the cessation of puberty and new integrated edges are seen [19], similar to what has been documented in the mandible.

Modularization of the alveolus and the dentition from the other portions of the mandible are a basic mammalian bauplan [66], one which is extraordinarily ancient [68]. Given this, how then does the pattern of integration in the modern human mandible compare to integration in other mammalian mandibles?

This same dissociation of the alveolar region from the ascending ramus was also noted in the mandibles of mice; the mouse mandible showed modularization between these two regions, albeit an incomplete form of modularization, indicating that a relationship still exists between these two areas $[51,52]$. Modularity in the mouse mandible is a matter of degrees, not absolutes [52], and because the mandible must function as a whole, the minor degree of interaction between modules seen in the mouse mandible is expected and required [51]. This functioning of distinct modules within the overall bauplan of the mandible can also be seen in the modern human mandible. Mandibular length generally possesses integrated edges with the other mandibular measurements, even when those other measurements come from different modules.

The mouse mandible is also characterized by strong modularity between the anterior and posterior portions of the mandible [51], likely the result of the anterior-posterior chewing pattern seen in mice [50]. It is possible that the increasing modularization seen in the edges involving anterior mandibular height is caused by a similar separation of the anterior and posterior portions of the human mandible (a similar anterior-posterior dichotomy is seen in baboon mandibles [50]).

Baboon mandibles have been shown to possess the same generalized mammalian mandibular integration bauplan seen in mice and modern humans. Like those two groups, baboons are characterized by modularity between the alveolar region and the ascending ramus, as well as modularity between the anterior (incisor) and posterior (molar) alveolar regions. Baboons are distinct from the mouse pattern of mandibular integration in having modules within the ascending ramus, specifically in the coronoid and alveolar processes [50]. If this increased number of distinct modules within the ascending ramus is in fact caused by the mediallateral chewing motion of baboons [50], then further quantification of the modern human upper ascending ramus may yield similar results, given that modern humans and baboons share a similar chewing motion.

Daegling [53] reported on growth in the African ape mandible, portions of which dealt with integration and allow for a limited comparison between ape and modern human mandibles. He found that the growth in different regions is decoupled (modularized) to a very large degree in ape 
mandibles. This same result can be seen in the modern human mandible, where those edges that are modularized tend to be very strongly modularized (Tables 2-4). Also, in African apes, it appears that corpus growth is regulated mainly by the need to house the dentition, whereas growth in the ascending ramus tends to be driven by the musculature [53].

The modern human mandible, during the juvenile and adolescent stages, shares an integration pattern with African apes of modularity between regions of the ascending ramus and corpus [53]. In the adult stage, modern humans are still characterized by modularity between the ascending ramus and the alveolar portions of the corpus, a pattern shared not only by African apes, but by mice [50-52] and baboons as well [50]. These similarities support the hypothesis that modern human mandibles are following a mammalianwide pattern of mandibular integration, one that is well established and largely invariant.

While the mammalian mandibles appear to share a similar pattern of integration, mammalian crania do not. Modern humans and African apes are characterized by different patterns of integration in the cranium, both as adults [13, $18,19]$ and during ontogeny $[12,19]$, with strong differences in the facial skeleton related to lower facial prognathism and upper facial height $[18,19]$. The strong differences in the pattern of integration in the cranium coupled with the strong similarities in the pattern of integration in the mandible between apes and modern humans (in the midsagittal plane) are supportive of the mandible being a more passive structure in the evolutionary history of the African hominoid skull. Instead, differences in the mandibles of modern humans relative to other hominoids are probably secondary consequences of the changes seen in the cranium, particularly the decreased prognathism and dentognathic size $[55,56]$.

The results of these studies would seem to indicate that growth and integration in the mandible follow Moss's functional matrix hypothesis, which states that if different regions of a system perform different functions, there is no necessary relationship between size, shape, spatial relationship $[3,74]$ or even patterns of integration of said regions. Even in a confined morphological unit such as the mandible, there is room for flexibility in various regions at different developmental stages depending upon the function required of those regions at that time.

\section{Conclusion}

The pattern of integration in the modern human mandible during ontogeny is dynamic. The overall trend is one of increasing modularity of the alveolar portion of mandible from the corpus and ascending ramus from childhood to adulthood, with those latter regions remaining integrated throughout ontogeny. Puberty is marked by a sudden increase in the amount of modularity in the mandible (a condition that is mirrored in the modern human cranium at the same time), which may be the result of a need to increase the adaptability of this region during a time of rapid facial growth.
The overall pattern of integration seems reflective of the dual nature of the hominin mandible: to house dentition and serve as muscle attachment sites. Regions of the mandible which serve these different functions arise from different embryological origins [66]; the modularization of these two regions from one another (ascending ramus and the alveolar portions of the mandible) throughout the development of the mandible is both expected and seen in this analysis.

The areas of the mandible concerned with the attachment of the chewing muscles remain relatively stable during ontogeny with respect to the pattern of integration. The major changes in the pattern of integration in the mandible occur in those regions associated with the dentition. Changes in the pattern of integration in the alveolar regions of the mandible are likely reflective of the differing emphasis placed on the dentition at different stages of growth. Early on in development there exists a greater need for enough space to house all the erupted deciduous dentition as well as the developing permanent dentition; this is reflected in the modularization of the superior portion of the mandible. In adulthood, there is a greater need for interaction between the maxillary and mandibular dentition, seen in the modularization of the anterior corpus region.

The human mandible has a pattern of integration similar to what has been documented in the African apes, a separation of the alveolar and ramus regions [53]; this pattern follows the embryological origins of these regions in the mammalian mandible as a whole [66], and these origins began early in mammalian evolution [68]. The hominin cranium must house the brain, the sight, smell and hearing organs, as well as aspects of the respiratory system; the majority of which must function for survival. The mandible only needs enough muscle function and occlusal contact to allow minimal mastication. Given the differences in cranial integration between humans and chimpanzees during ontogeny $[12,13,18,19]$, and the similarities in mandibular integration, it seems that the mandible plays "follow the leader" with the cranium during hominin evolution.

\section{Acknowledgments}

The author would like to thank Tom Southard and the Department of Orthodontics at the University of Iowa for their help in gaining access to the Iowa Facial Growth Study. He would also like to thank Hannah E. Marsh and Steven F. Miller for their insightful comments on this paper.

\section{References}

[1] F. Weidenreich, "The extremity bones of sinanthropus pekinensis," Palaeontologica Sinica New Series D, vol. 5, pp. 1150, 1941.

[2] F. Weidenreich, "The skull of sinanthropus pekinensis: a comparative study of a primitive hominid skull," Palaeontologica Sinica New Series D, vol. 10, pp. 1-485, 1943.

[3] M. L. Moss, "The functional matrix," in Vistas in Orthodontics, B. Kraus and R. Reidel, Eds., pp. 85-98, Lea and Febiger, Philadelphia, Pa, USA, 1962. 
[4] M. L. Moss and L. Salentijn, "The capsular matrix," American Journal of Orthodontics, vol. 56, no. 5, pp. 474-490, 1969.

[5] R. R. Ackermann and J. M. Cheverud, "Phenotypic covariance structure in tamarins (genus Saguinus): a comparison of variation patterns using matrix correlation and common principal component analysis," American Journal of Physical Anthropology, vol. 111, no. 4, pp. 489-501, 2000.

[6] J. M. Cheverud, "Morphological integration in the saddleback tamarin (Saguinus fuscicollis) cranium," American Naturalist, vol. 145, no. 1, pp. 63-89, 1995.

[7] J. M. Cheverud, "Spatial-analysis in morphology illustrated by rhesus macaque cranial growth and integration," American Journal of Physical Anthropology, vol. 75, pp. 195-196, 1988.

[8] J. M. Cheverud, "Phenotypic, genetic, and environmental morphological integration in the cranium," Evolution, vol. 36, pp. 499-516, 1982.

[9] J. M. Cheverud, "Quantitative genetic analysis of cranial morphology in the cotton-top (Saguinus oedipus) and saddle-back (S. fuscicollis) tamarins," Journal of Evolutionary Biology, vol. 9, no. 1, pp. 5-42, 1996.

[10] G. Marroig and J. M. Cheverud, "Cranial evolution in sakis (Pithecia, Platyrrhini) I: interspecific differentiation and allometric patterns," American Journal of Physical Anthropology, vol. 125, no. 3, pp. 266-278, 2004.

[11] G. Marroig and J. M. Cheverud, "A comparison of phenotypic variation and covariation patterns and the role of phylogeny, ecology, and ontogeny during cranial evolution of New World Monkeys," Evolution, vol. 55, no. 12, pp. 2576-2600, 2001.

[12] R. R. Ackermann, "Ontogenetic integration of the hominoid face," Journal of Human Evolution, vol. 48, no. 2, pp. 175-197, 2005.

[13] R. R. Ackermann, "Patterns of covariation in the hominoid craniofacial skeleton: implications for paleoantropological models," Journal of Human Evolution, vol. 43, no. 2, pp. 167 $187,2002$.

[14] D. S. Strait, "Integration, phylogeny, and the hominid cranial base," American Journal of Physical Anthropology, vol. 114, no. 4, pp. 273-297, 2001.

[15] F. L. Bookstein, P. Gunz, P. Mittercker, H. Prossinger, K. Schæfer, and H. Seidler, "Cranial integration in Homo: singular warps analysis of the midsagittal plane in ontogeny and evolution," Journal of Human Evolution, vol. 44, no. 2, pp. 167$187,2003$.

[16] D. E. Lieberman, G. E. Krovitz, and B. Mcbratney-Owen, "Testing hypotheses about tinkering in the fossil record: the case of the human skull," Journal of Experimental Zoology Part B, vol. 302, no. 3, pp. 284-301, 2004.

[17] D. E. Lieberman, B. M. McBratney, and G. Krovitz, "The evolution and development of cranial form in Homo sapiens," Proceedings of the National Academy of Sciences of the United States of America, vol. 99, no. 3, pp. 1134-1139, 2002.

[18] J. M. Polanski and R. G. Franciscus, "Patterns of craniofacial integration in extant Homo, Pan, and Gorilla," American Journal of Physical Anthropology, vol. 131, no. 1, pp. 38-49, 2006.

[19] J. M. Polanski, Morphological Integration of the Cranium during Ontogeny in Homo Sapiens and Pan Troglodytes, University of Iowa, Iowa City, Iowa, USA, 2009.

[20] N. G. Leakey, C. S. Feibel, I. McDougall, and A. Walker, "New four-million-year-old hominid species from Kanapoi and Allia Bay, Kenya," Nature, vol. 376, no. 6541, pp. 565-571, 1995.

[21] M. D. Leakey, R. L. Hay, G. H. Curtis, R. E. Drake, M. K. Jackes, and T. D. White, "Fossil hominids from the Laetolil Beds," Nature, vol. 262, no. 5568, pp. 460-466, 1976.
[22] M. Brunet, A. Beauvilain, Y. Coppens, E. Heintz, A. H. E. Moutaye, and D. Pilbeam, "The first australopithecine 2,500 kilometres west of the Rift Valley (Chad)," Nature, vol. 378, no. 6553, pp. 273-275, 1995.

[23] R. Broom, "Another new type of fossil ape-man," Nature, vol. 163, no. 4132, p. 57, 1949.

[24] C. P. Groves and V. Mazak, "An approach to the taxonomy of the hominidae: gracile villafranchian hominids of africa," Casopis pro Mineralogii a Geologii, vol. 20, pp. 225-247, 1975.

[25] O. Schoetensack, Der unterkiefer des homo heidelbergensis aus den sanden von mauer bei heidelberg, Wilhelm Engleman, Leipzig, Germany, 1908.

[26] D. J. Daegling and W. L. Hylander, "Biomechanics of torsion in the human mandible," American Journal of Physical Anthropology, vol. 105, no. 1, pp. 73-87, 1998.

[27] W. L. Hylander, "Mandibular function and biomechanical stress and scaling," Integrative and Comparative Biology, vol. 25, no. 2, pp. 315-330, 1985.

[28] W. L. Hylander and K. R. Johnson, "Jaw muscle function and wishboning of the mandible during mastication in macaques and baboons," American Journal of Physical Anthropology, vol. 94, no. 4, pp. 523-547, 1994.

[29] W. L. Hylander, K. R. Johnson, and A. W. Crompton, "Loading patterns and jaw movements during mastication in Macaca fascicularis: a bone-strain, electromyographic, and cineradiographic analysis," American Journal of Physical Anthropology, vol. 72, no. 3, pp. 287-314, 1987.

[30] W. L. Hylander, M. J. Ravosa, C. F. Ross, and K. R. Johnson, "Mandibular corpus strain in primates: further evidence for a functional link between symphyseal fusion and jaw-adductor muscle force," American Journal of Physical Anthropology, vol. 107, no. 3, pp. 257-271, 1998.

[31] C. F. O’Connor, R. G. Franciscus, and N. E. Holton, “Bite force production capability and efficiency in neandertals and modern humans," American Journal of Physical Anthropology, vol. 127, no. 2, pp. 129-151, 2005.

[32] W. L. Hylander, "Stress and strain in the mandibular symphysis of primates: a test of competing hypotheses," American Journal of Physical Anthropology, vol. 64, no. 1, pp. 1-46, 1984.

[33] D. J. Daegling, "Biomechanical scaling of the hominoid mandibular symphysis," Journal of Morphology, vol. 250, no. 1, pp. 12-23, 2001.

[34] S. D. Dobson and E. Trinkaus, "Cross-sectional geometry and morphology of the mandibular symphysis in Middle and Late Pleistocene Homo," Journal of Human Evolution, vol. 43, no. 1, pp. 67-87, 2002.

[35] A. Rosas, "A gradient of size and shape for the Atapuerca sample and Middle Pleistocene hominid variability," Journal of Human Evolution, vol. 33, no. 2-3, pp. 319-331, 1997.

[36] H. Fukase, "Functional significance of bone distribution in the human mandibular symphysis," Anthropological Science, vol. 115, no. 1, pp. 55-62, 2007.

[37] W. L. Hylander, M. J. Ravosa, C. F. Ross, C. E. Wall, and K. R. Johnson, "Symphyseal fusion and jaw-adductor muscle force: an EMG study," American Journal of Physical Anthropology, vol. 112, no. 4, pp. 469-492, 2000.

[38] V. H. Stefan and E. Trinkaus, "Discrete trait and dental morphometric affinities of the Tabun 2 mandible," Journal of Human Evolution, vol. 34, no. 5, pp. 443-468, 1998.

[39] A. B. Taylor, "Masticatory form and function in the African apes," American Journal of Physical Anthropology, vol. 117, no. 2, pp. 133-156, 2002. 
[40] A. B. Taylor, "Diet and mandibular morphology in African apes," International Journal of Primatology, vol. 27, no. 1, pp. 181-201, 2006.

[41] A. B. Taylor, "Feeding behavior, diet, and the functional consequences of jaw form in orangutans, with implications for the evolution of Pongo," Journal of Human Evolution, vol. 50, no. 4, pp. 377-393, 2006.

[42] M. J. Ravosa, "Size and scaling in the mandible of living and extinct apes," Folia Primatologica, vol. 71, no. 5, pp. 305-322, 2000.

[43] J. T. Robinson, "The genera and species of the Australopithecinae," American Journal of Physical Anthropology, vol. 12, no. 2, pp. 181-200, 1954.

[44] J. T. Robinson, Early Hominid Posture and Locomotion, University of Chicago Press, Chicago, Ill, USA, 1972.

[45] J. T. Robinson, "Prehominid dentition and hominid evolution," Evolution, vol. 8, pp. 324-334, 1954.

[46] R. Kay and F. Grine, "Tooth morphology, wear and diet in australopithecus and paranthropus from southern africa," in Evolutionary History of the "Robust" Australopithecines, F. Grine, Ed., pp. 427-447, Aldine de Gruyter, New York, NY, USA, 1988.

[47] R. F. Kay, "Dental evidence for the diet of Australopithecus," Annual Review of Anthropology, vol. 14, pp. 315-341, 1985.

[48] B. A. Wood, "Tooth size and shape and their relevance to studies of hominid evolution," Philosophical Transactions of the Royal Society B, vol. 292, no. 1057, pp. 65-76, 1981.

[49] A. Walker, "Diet and teeth. Dietary hypotheses and human evolution," Philosophical Transactions of the Royal Society B, vol. 292, no. 1057, pp. 57-64, 1981.

[50] K. E. Willmore, C. C. Roseman, J. Rogers, J. M. Cheverud, and J. T. Richtsmeier, "Comparison of mandibular phenotypic and genetic integration between baboon and mouse," Evolutionary Biology, vol. 36, no. 1, pp. 19-36, 2009.

[51] C. P. Klingenberg, K. Mebus, and J. C. Auffray, “Developmental integration in a complex morphological structure: how distinct are the modules in the mouse mandible?" Evolution and Development, vol. 5, no. 5, pp. 522-531, 2003.

[52] C. P. Klingenberg, L. J. Leamy, and J. M. Cheverud, "Integration and modularity of quantitative trait locus effects on geometric shape in the mouse mandible," Genetics, vol. 166, no. 4, pp. 1909-1921, 2004.

[53] D. J. Daegling, "Growth in the mandibles of African apes," Journal of Human Evolution, vol. 30, no. 4, pp. 315-341, 1996.

[54] M. H. Wolpoff, Paleoanthropology, McGraw-Hill, Boston, Mass, USA, 1999.

[55] R. G. Franciscus and E. Trinkaus, "Determinants of retromolar space presence in Pleistocene Homo mandibles," Journal of Human Evolution, vol. 28, no. 6, pp. 577-595, 1995.

[56] E. Trinkaus, "Neandertal faces were not long; modern human faces are short," Proceedings of the National Academy of Sciences of the United States of America, vol. 100, no. 14, pp. 8142-8145, 2003.

[57] D. E. Lieberman, “Testing hypotheses about recent human evolution from skulls_-integrating morphology, function, development, and phylogeny," Current Anthropology, vol. 36, pp. 159-197, 1995.

[58] H. V. Meredith and J. M. Chadha, "A roentgenographic study of change in head height during childhood and adolescence," Human Biology, vol. 34, pp. 299-319, 1962.

[59] M. L. Zelditch, F. L. Bookstein, and B. L. Lundrigan, "Ontogeny of integrated skull growth in the cotton rat sigmodon fulviventer," Evolution, vol. 46, pp. 1164-1180, 1992.
[60] W. M. Merow and B. H. Broadbent, "Cephalometrics," in Facial Growth, D. H. Enlow, Ed., pp. 346-395, W.B. Saunders, Philadelphia, Pa, USA, 1990.

[61] B. H. Broadbent, B. H. Broadbent Jr., and W. H. Golden, Bolton Standards of Dentofacial Developmental Growth, C.V. Mosby, St. Louis, Mo, USA, 1975.

[62] J. F. Pachut and M. M. Fisherkeller, "Changes in colonial development, intraspecific heterochrony, morphological integration, and character heritabilities in two populations of the bryozoan species Batostoma jamesi from the kope formation (Upper Ordovician, Cincinnatian)," Journal of Paleontology, vol. 76, no. 2, pp. 197-210, 2002.

[63] W. S. Armbruster, V. S. Di Stilio, J. D. Tuxill, T. C. Flores, and J. L. Velásquez Runk, "Covariance and decoupling of floral and vegetative traits in nine neotropical plants: a re-evaluation of Berg's correlation-pleiades concept," American Journal of Botany, vol. 86, no. 1, pp. 39-55, 1999.

[64] R. A. Adams, "Evolutionary implications of developmental and functional integration in bat wings," Journal of Zoology, vol. 246, no. 2, pp. 165-174, 1998.

[65] P. M. Magwene, "New tools for studying integration and modularity," Evolution, vol. 55, no. 9, pp. 1734-1745, 2001.

[66] W. R. Atchley and B. K. Hall, "A model for development and evolution of complex morphological structures," Biological Reviews of the Cambridge Philosophical Society, vol. 66, no. 2, pp. 101-157, 1991.

[67] J. M. Cheverud, "Developmental integration and the evolution of pleiotropyl," American Zoologist, vol. 36, no. 1, pp. 44-50, 1996.

[68] M. M. Smith and B. K. Hall, "Development and evolutionary origins of vertebrate skeletogenic and odontogenic tissues," Biological Reviews of the Cambridge Philosophical Society, vol. 65, no. 3, pp. 277-373, 1990.

[69] D. H. Ubelaker, Human Skeletal Remains: Excavation, Analysis, Intepretation, Taraxacum, Washington, DC, USA, 1989.

[70] R. E. Bradley, "The relationship between eruption, calcification, and crowding of certain mandibular teeth," Angle Orthodontist, vol. 41, pp. 230-236, 1961.

[71] J. W. Osborn, "Morphogenetic gradients: fields versus clones," in Development, Function and Evolution of Teeth, P. M. Butler and K. A. Joysey, Eds., pp. 171-201, Academic Press, London, UK, 1978.

[72] M. C. Dean and A. D. Beynon, "Tooth crown heights, tooth wear, sexual dimorphism and jaw growth in hominoids," Zeitschrift für Morphologie und Anthropologie, vol. 78, no. 3, pp. 425-440, 1991.

[73] R. L. Tompkins, "Human population variability in relative dental development," American Journal of Physical Anthropology, vol. 99, no. 1, pp. 79-102, 1996.

[74] M. L. Moss, "Functional cranial analysis of mammalian mandibular ramal morphology," Acta Anatomica, vol. 71, no. 3, pp. 423-447, 1968. 

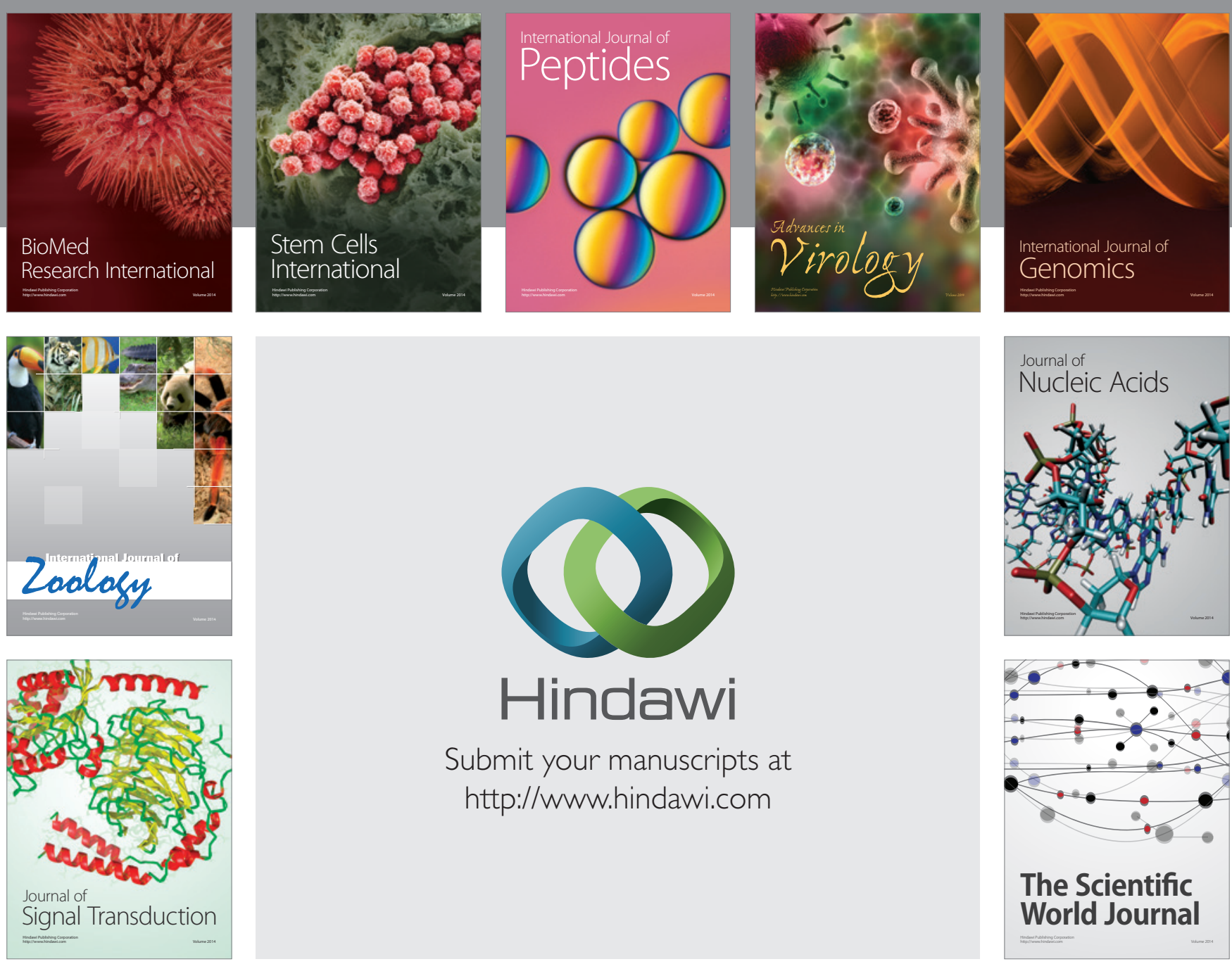

Submit your manuscripts at

http://www.hindawi.com
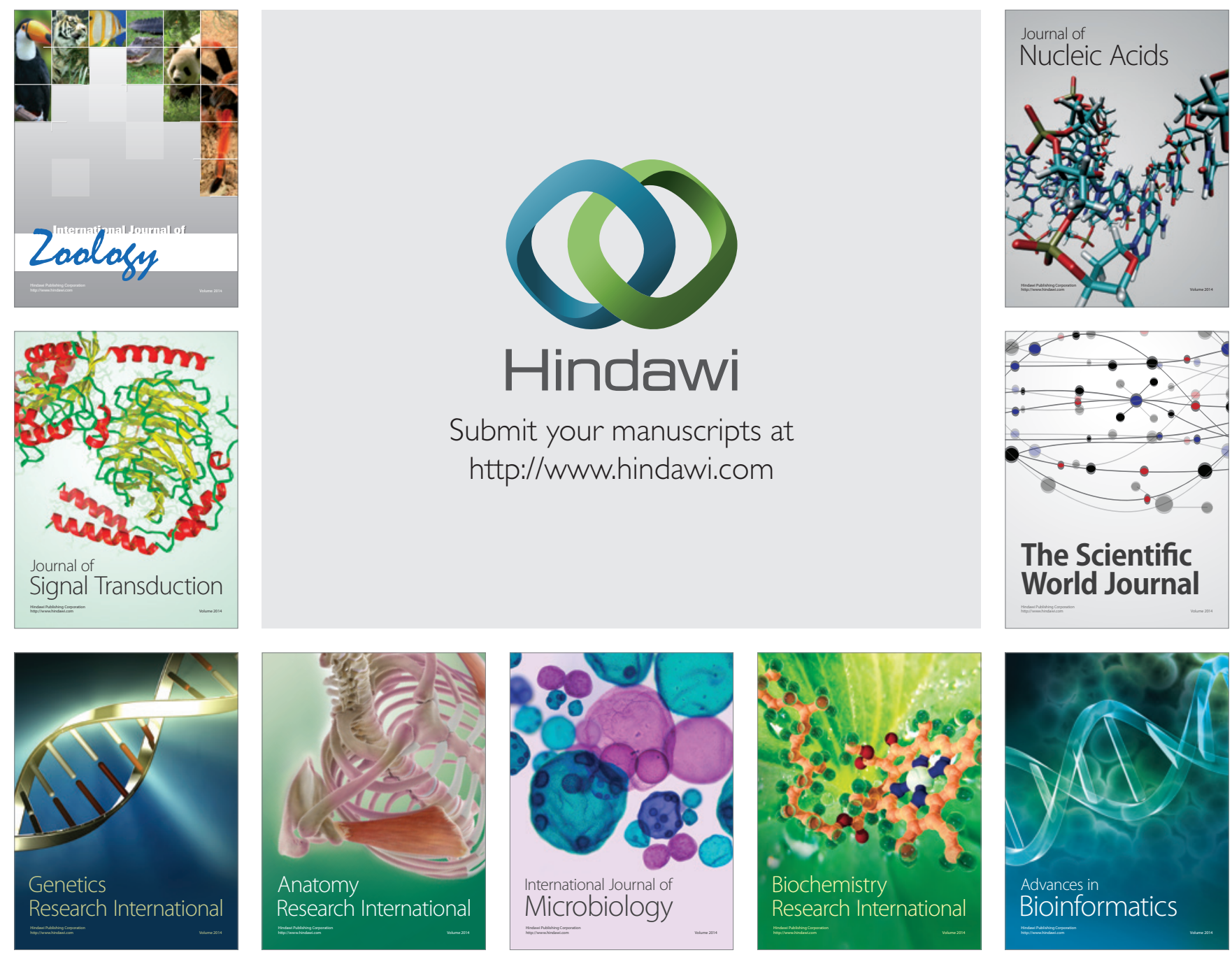

The Scientific World Journal
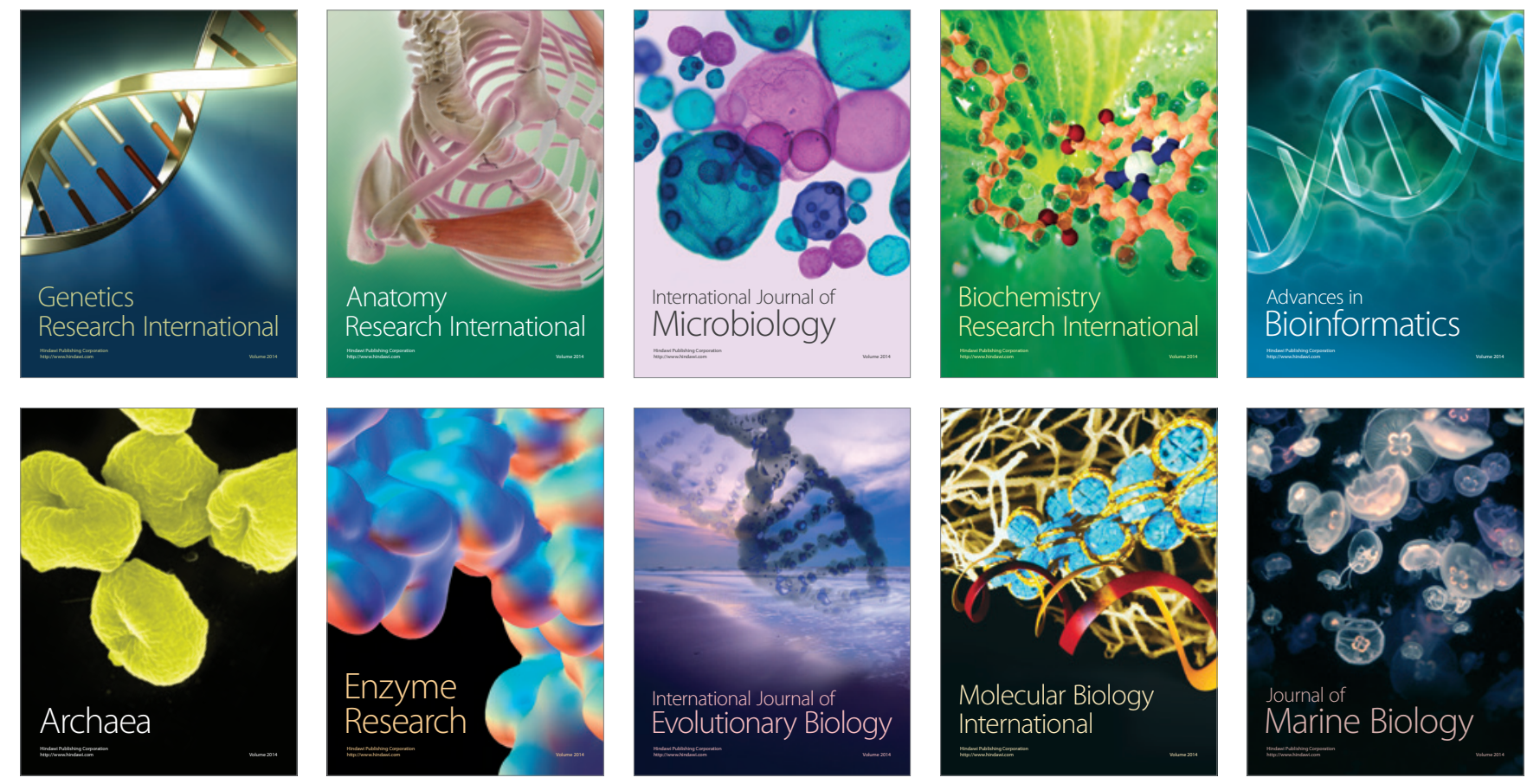